\title{
Analyse du profil protéique chez des vaches zébus Gobra artificiellement inséminées au Sénégal
}

\author{
Mohamed Moctar MOUICHE MOULIOM ${ }^{1,2,3^{*}}$, Adama SOW ${ }^{2}$, Miguiri KALANDI ${ }^{2}$, \\ Serge Eugene MPOUAM ${ }^{1}$, Georges Anicet OUEDRAOGO ${ }^{3}$ et \\ Germain Jérôme SAWADOGO ${ }^{2}$ \\ ${ }^{1}$ Ecole des Sciences et de Médecine Vétérinaire (ESMV), Université de Ngaoundéré. BP 454 Ngaoundéré, \\ Cameroun. \\ ${ }^{2}$ Ecole Inter-Etats des Sciences et Médecine Vétérinaires (EISMV), Laboratoire d'Endocrinologie et de \\ Radioimmunologie. BP 5077, Dakar Fann, Sénégal. \\ ${ }^{3}$ Université Polytechnique de Bobo-Dioulasso, Laboratoire d'Enseignement et de Recherche en Santé et \\ Biotechnologie Animales, 01 BP 1091, Bobo-Dioulasso 01, Burkina Faso. \\ *Auteur correspondant : mouichemoctar@yahoo.fr
}

\section{RESUME}

L'objectif de cette étude a été d'analyser le profil protéique des vaches artificiellement inséminées en fonction de leur statut physiologique. Des prélèvements de sang ont été effectués sur 81 vaches inséminées de race zébu Gobra à $J_{0}, J_{21}$ et $J_{35}$ post insémination. La protéinémie a été évaluée à $J_{35}$ post insémination. L'électrophorèse des protéines sériques a été mise en œuvre sur tous les échantillons. Le diagnostic de gestation a été réalisé par dosage de la progestérone, des protéines associées à la gestation et la palpation transrectale à $\mathrm{J}_{60}$ après l'insémination artificielle (IA). Au total, 47\% des vaches étaient gestantes, $26 \%$ ont présentées des mortalités embryonnaires tardives (MET) et $27 \%$ étaient non gestantes à $\mathrm{J}_{60}$ après l'IA. Le rapport albumine sur globuline $(\mathrm{A} / \mathrm{G})$ était significativement différent $(\mathrm{p}<0,05)$ entre les vaches non gestantes et celles ayant subi des pertes embryonnaires. Chez les vaches ayant avorté, les fractions de gammaglobulines étaient significativement plus élevées que chez les vaches gestantes $(\mathrm{p}<0,05)$. Par conséquent, des causes infectieuses seraient responsables de ces pertes embryonnaires. Un accent particulier doit être mis ces MET en vu d'élucider les causes et limiter les pertes économiques car, en absence de subvention, le coût de l'IA reste élevé pour l'éleveur africain.

(c) 2013 International Formulae Group. All rights reserved.

Mots clés: Profil protéique, électrophorèse, insémination artificielle, zébu Gobra, Sénégal.

\section{INTRODUCTION}

L'amélioration de la fertilité demeure l'un des objectifs prioritaires pour optimiser le potentiel de reproduction et donc de production en élevage bovin. L'analyse des résultats sur l'insémination artificielle (IA) en
Afrique subsaharienne a montré de faible taux de réussite. Plusieurs facteurs sont à l'origine de ces faibles taux de réussite de l'IA; notamment la non maîtrise des paramètres de reproduction chez la vache, l'alimentation, les facteurs sanitaires, environnementaux et 
surtout les mortalités embryonnaires (ME) et les avortements (Kouamo et al., 2009; Kouamo et al., 2010).

Les proportions et les causes de ces ME demeurent encore mal étudiées au niveau du Sénégal. Les causes des pertes embryonnaires varient en fonction de la période ou du stade de la gestation. Après l'insémination, la ME est reconnue comme l'une des causes majeures de l'échec de reproduction en élevage (Dunne et al., 2000 ; Inskeep and Dailey, 2005; Santos et al., 2009). Elle se traduit par un nombre moins important de nouveau-nés, une perte en lait pour les élevages laitiers, un progrès génétique ralenti et une perte financière significative de revenu pour l'éleveur (Dunne et al., 2000). Plusieurs facteurs étiologiques ont été répertoriés comme responsables des ME principalement : les facteurs gamétiques, embryonnaires, parentaux, climatiques, alimentaires et biologiques (pathologies de la reproduction) (Romano et al., 2007 ; Bajaj and Sharma, 2011).

L'objectif général de cette étude était d'analyser le profil protéique des vaches inséminées en fonction de leur statut physiologique (gestantes, non gestantes et mortalités embryonnaires). Il s'agissait d'évaluer les causes de ME chez les bovins par l'exploration des indicateurs métaboliques de l'efficacité du système de défense. L'électrophorèse des protéines sériques est actuellement une analyse très utilisée en biologie clinique (Alberghina et al., 2011). Elle permet ainsi d'explorer ces indicateurs métaboliques de l'efficacité du système de défense des animaux (globulines totales). Les proportions des différentes fractions protéiques (albumine, $\alpha$-globuline, $\beta$ globuline et $\gamma$-globuline) ont un intérêt diagnostic différent en fonction de leur sens de variation.

\section{MATERIEL ET METHODES Milieu d'étude}

L'étude a été réalisée en milieu rural et urbain des régions de Dakar et de Thiès, situées en zone sahélienne entre les isohyètes 400 et $600 \mathrm{~mm}$ (Figure 1). Le climat, de type sahélien, est caractérisé par une saison des pluies d'une durée variable (juillet - octobre) et une saison sèche (novembre - juin). Les races bovines locales exploitées dans ces régions sont par ordre d'importance le zébu Gobra (Bos indicus), le taurin N'dama (Bos taurus africanus), le zébu Maure et le Djakoré (Gobra*N'dama) (Kouamo et al., 2009)..

\section{Échantillonnage des vaches}

L'échantillon d'étude a été composé de 81 vaches de races Gobra appartenant à 25 élevages. Elles ont été inséminées dans le cadre du Programme National d'Insémination Artificielle (PNIA). Les vaches étaient composées de génisses ou de vaches ayant plus de 3 mois de période post-partum. La note d'état corporel (NEC) de toutes les vaches était comprise entre 2,5 - 4 et l'âge moyen de $6 \pm 2$ ans. La note d'état corporel a été évaluée sur une grille de 0 à 5 appliquée aux zébus d'Afrique de l'Ouest (Vall and Bayala, 2004). Les animaux sélectionnés ont été identifiés à l'aide des boucles auriculaires et toutes les informations les concernant ont été enregistrées sur des fiches individuelles.

Les vaches étaient conduites selon le mode extensif ou semi extensif, caractérisé par la recherche du pâturage naturel pendant la journée et une supplémentation, au retour dans les enclos au coucher du soleil, à l'aide de l'aliment bétail commercial avant la traite. Les animaux s'abreuvaient dans des mares au pâturage.

Les conditions imposées par le programme d'insémination étaient la pratique de la stabulation pour les animaux 
sélectionnés, la capacité des éleveurs à assurer la complémentation et en cas de nécessité l'apport de soins (déparasitage, vaccination...) aux animaux. Les inséminations ont été réalisées sur chaleurs induites avec un protocole associant la spirale vaginale $\left(\mathrm{PRID}^{\mathrm{ND}}\right)$ à la prostaglandine $\mathrm{F}_{2 \alpha}$ et à la Pregnant Mare Serum Gonadotropin (PMSG).

\section{Prélèvement de sang}

De mai 2007 à mars 2008, des prélèvements de sang ont été réalisés à $\mathrm{J}_{0}, \mathrm{~J}_{21}$, et $\mathbf{J}_{35}$ après l'insémination artificielle (IA) par ponction de la veine jugulaire dans des tubes à héparines secs puis centrifugés (3500 tours par minutes pendant 20 minutes) pour obtenir le plasma et le sérum qui ont été conservés à $20^{\circ} \mathrm{C}$ jusqu'à l'analyse.

\section{Diagnostic de gestation et des mortalités embryonnaires}

Les concentrations plasmatiques de PAGs $\left(\mathbf{J}_{0}\right.$ et $\left.\mathbf{J}_{35}\right)$ et de progestérone $\left(\mathbf{J}_{0}\right.$, et $\left.\mathbf{J}_{21}\right)$ ont été mesurées par dosage radioimmunologique (RIA) (Zoli et al., 1992; Sousa et al., 2003 ; Ponsart et al., 2007) pour déterminer l'état physiologique des femelles (gestantes, non gestantes ou mortalités embryonnaires). La palpation transrectale a été réalisée à $\mathrm{J}_{60}$ après l'IA pour la confirmation de l'état gravidique des animaux. L'association entre les différents dosages (PAGs et progestérone) et le diagnostic par palpation transrectale conduit à l'obtention de différents statuts physiologiques à $\mathrm{J}_{60}$ après l'IA.

\section{Dosage des protéines totales et électrophorèse}

La protéinémie a été évaluée chez les animaux à $\mathrm{J}_{35}$ post insémination par dosage colorimétrique à l'aide d'un kit fourni par le Laboratoire BIOSYSTEMS $^{\circledR}$ S.A (Barcelone, Espagne). Le principe du dosage est la méthode de Biuret en milieu alcalin (Tietz,
1994). Les densités optiques ont été lues à l'aide d'un spectrophotomètre d'absorption UV (BIOSYSTEMS ${ }^{\circledR}$ BTS-310).

L'électrophorèse en zone sur gel d'agarose (Alberghina et al., 2011) a été réalisée sur chaque échantillon en utilisant le kit d'électrophorèse Hydragel protéine K20 SEBIA $^{\circledR}$ (France). Le profil électrophorétique a été déterminé selon les recommandations du fabricant. Un volume de $10 \mu \mathrm{l}$ de sérum non dilué a été appliqué sur le gel avec le peigne fourni. Le gel a été placé dans une chambre de migration $\left(\mathrm{SEBIA}^{\circledR}{ }^{\circledR}\right.$, France) et le tampon utilisé a été le tampon tris-barbital $(\mathrm{pH}=8,5)$. La différence de potentiel utilisée pour la migration était de 90 volts pendant une durée de 22 minutes. L'intensité du courant est de \pm $12 \mathrm{~mA}$ par gel.

Apres la migration, le gel a été fixé et séché dans un incubateur sécheur (IS 80 SEBIA $^{\circledR}$ ) à $80{ }^{\circ} \mathrm{C}$ pendant au moins 10 minutes, coloré dans une solution amidoswarz (acide acétique) pendant 4 minutes et décoloré dans trois bains successifs de solution de décoloration jusqu'à obtention d'un fond clair. Les protéinogrammes ont été analysés à l'aide d'un densitomètre (HYRYS SEBIA ${ }^{\circledR}$, France) à $570 \mathrm{~nm}$. Le profil électrophorétique représente les différentes fractions suivantes : albumine, $\alpha 1$-globuline, $\alpha 2$-globuline, $\beta$ globuline, et $\gamma$-globuline. Le pourcentage relatif de chaque fraction protéique a été calculé à l'aide d'un densitomètre.

\section{Analyses statistiques}

Les données collectées ont été saisies et traitées par le logiciel SPSS $12.0^{\circledR}$. Les résultats ont été exprimés en moyenne \pm écart type. La comparaison des moyennes entre groupe a été effectuée par ANOVA ou le test de Student (t-test). Les différences sont considérées significatives au seuil de probabilité $\mathrm{p}<0,05$. 


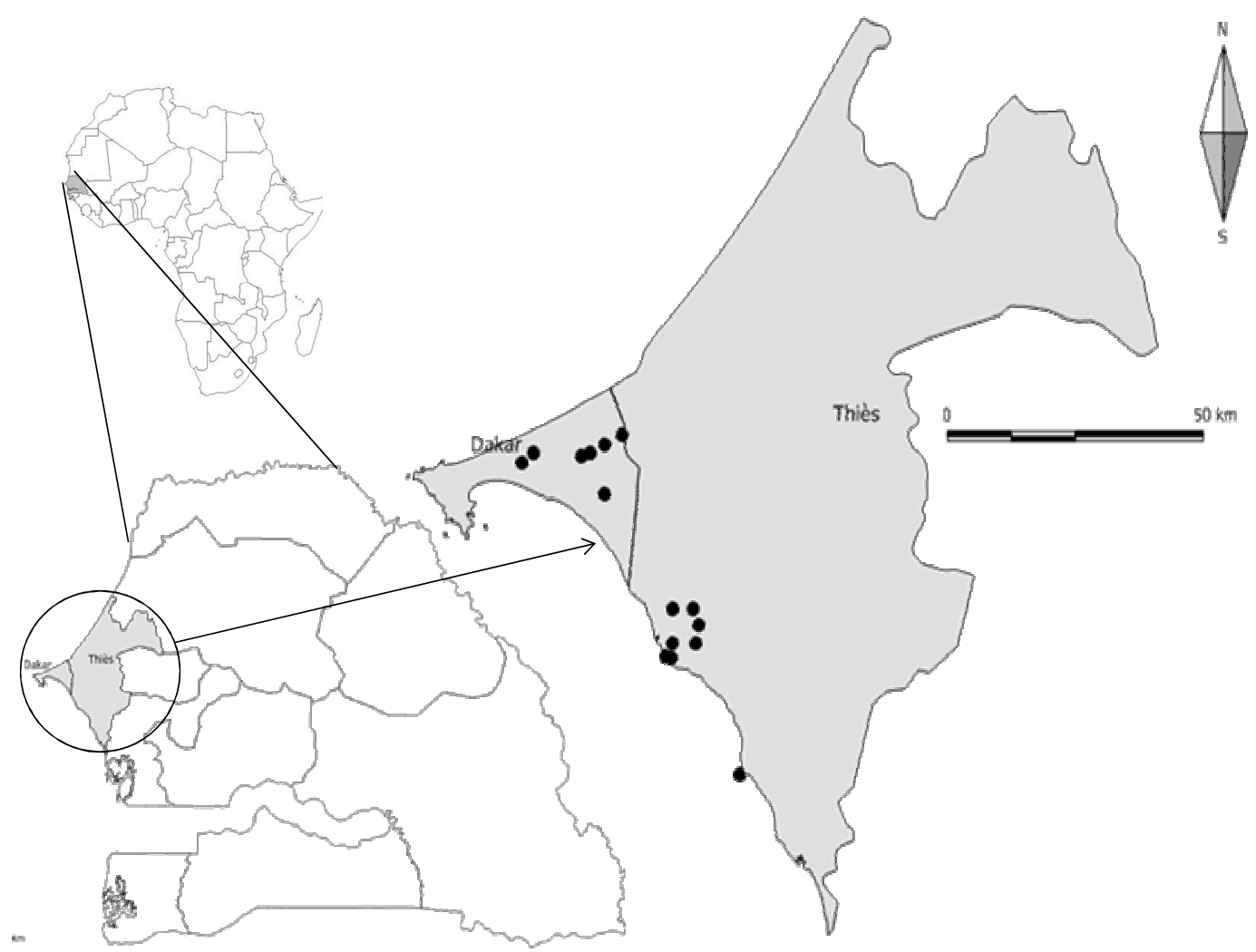

Figure 1: Carte du Sénégal montrant le site d'étude et les zones où les prélèvements ont été effectués.

\section{RESULTATS}

Taux de réussite de l'insémination

Le diagnostic de gestation par palpation transrectale $\left(\mathrm{J}_{60}\right)$ et par dosage de la progestérone $\left(\mathrm{J}_{21}\right)$ et des PAGs $\left(\mathrm{J}_{35}\right)$ a permis de classer les vaches inséminées en gestantes, non gestantes et MET (Tableau 1). Sur 81 vaches inséminées, 38 (soit 47\%) ont été gestantes, $21(26 \%)$ ont avorté (MET) et 22 (27\%) étaient non gestantes à $\mathrm{J}_{60}$ après IA. Sur 21 vaches ayant présenté une perte embryonnaire, 11 ont été observées entre $\mathrm{J}_{21}$ $\mathrm{J}_{35}$ et 10 entre $\mathrm{J}_{35}-\mathrm{J}_{60}$.

\section{Profil protéique chez les vaches}

Le Tableau 2 présente les moyennes des fractions protéiques obtenues après électrophorèse chez les vaches en fonction de leur état physiologique.

Il existe une différence significative entre les concentrations de protéines totales en fonction de l'état physiologique $(\mathrm{p}<0,05)$. $\mathrm{La}$ fraction d'albumine représente en moyenne 46 - 50\% des protéines totales chez l'ensemble des vaches. La proportion en albumine a été significativement plus élevée chez les vaches non gestantes $(\mathrm{p}<0,05)$. Les vaches gestantes ont des proportions d' $\alpha 1$ globulines statistiquement plus élevées que celles ayant avorté $(\mathrm{p}<0,05)$.

Il y a eu une différence significative $(\mathrm{p}<0,05)$ entre les proportions de $\gamma$ globulines des vaches ayant subi des pertes embryonnaires $(33,53 \pm 5,67 \%)$ et celles des autres groupes. Le rapport albumine sur 
globuline $(\mathrm{A} / \mathrm{G})$ est significativement plus élevé $(\mathrm{p}<0,05)$ chez les vaches non gestantes $(1,09 \pm 0,17)$.

Le Tableau 3 présente les moyennes des fractions protéiques obtenues après électrophorèse chez le groupe des vaches ayant subi une MET en fonction de la période de survenu de la MET. Aucune différence significative n'a été observée entre les différentes fractions protéiques et la période de survenue des MET. L'analyse comparée du profil électrophorétique entre une vache ayant subi une MET et de celle de la vache non gestante montre une hypergammaglobulinémie (Figure 2).

Tableau 1: Correspondance entre différentes situations après insémination artificielle et les résultats des dosages de progestérone et PAGs.

\begin{tabular}{ccccc}
\hline Progestérone à $\mathbf{J}_{\mathbf{0}}$ & Progestérone à $\mathbf{J}_{\mathbf{2 1 - 2 4}}$ & PAGs à $\mathbf{J}_{\mathbf{3 5 - 3 5}}$ & ${\text { Gestation à } \mathbf{J}_{60}}$ & Diagnostic \\
\hline Faible & Faible & non détectée & non détectée & MEP ou NF \\
Faible & Élevée & non détectée & non détectée & MET \\
Faible & Élevée & détectée & non détectée & MET \\
Faible & Élevée & détectée & Détectée & Gestation \\
\hline PAGs: Protéines associées à la gestation: & MEP: & Mortalités & Embryonnaires précoces; & MET: Mortalités
\end{tabular}

PAGs : Protéines associées à la gestation; MEP : Mortalités Embryonnaires précoces ; MET: Mortalités Embryonnaires ; NF : Non Fécondation ; J : Jour

Tableau 2: Valeurs moyennes et proportions des différentes fractions protéiques chez les vaches Gobra en fonction de l'état physiologique.

\begin{tabular}{|c|c|c|c|c|}
\hline \multicolumn{2}{|l|}{$\begin{array}{l}\text { Paramètres } \\
\text { biochimiques }\end{array}$} & $\begin{array}{l}\text { MET } \\
(n=21)\end{array}$ & $\begin{array}{c}\text { Non gestantes } \\
(\mathbf{n}=\mathbf{2 2})\end{array}$ & $\begin{array}{c}\text { Gestantes } \\
(\mathbf{n}=38)\end{array}$ \\
\hline \multicolumn{2}{|c|}{ Protéines Totales g/l } & $81,48 \pm 14,9^{b}$ & $68,68 \pm 12,7^{\mathrm{a}}$ & $79,75 \pm 12,34^{b}$ \\
\hline \multirow{2}{*}{ Albumine } & $\mathrm{g} / \mathrm{l}$ & $35,93 \pm 5,37^{\mathrm{a}}$ & $37,98 \pm 6,06^{\mathrm{a}}$ & $37,63 \pm 7,15^{\mathrm{a}}$ \\
\hline & $\%$ & $46,08 \pm 6,08 \%^{\mathrm{a}}$ & $50,2 \pm 2,96^{\mathrm{b}}$ & $47,64 \pm 3,06^{\mathrm{c}}$ \\
\hline \multirow{2}{*}{$\alpha 1-G l o b u l i n e$} & $\mathrm{~g} / \mathrm{l}$ & $2,36 \pm 0,86^{\mathrm{a}}$ & $2,63 \pm 1,16^{\mathrm{a}}$ & $3,94 \pm 0,98^{b}$ \\
\hline & $\%$ & $2,76 \pm 1,01 \%{ }^{\mathrm{a}}$ & $3,25 \pm 1,97^{\mathrm{a}}$ & $4,4 \pm 1,36^{\mathrm{b}}$ \\
\hline \multirow{2}{*}{$\alpha 2-G l o b u l i n e$} & $\mathrm{~g} / \mathrm{l}$ & $8,02 \pm 1,61^{a}$ & $7,13 \pm 1,38^{\mathrm{a}}$ & $9,08 \pm 1,78^{\mathrm{a}}$ \\
\hline & $\%$ & $9,36 \pm 2,08 \%{ }^{a}$ & $9,9 \pm 1,56^{\mathrm{a}}$ & $10,56 \pm 3,27^{\mathrm{a}}$ \\
\hline \multirow{2}{*}{$\beta$-Globuline } & $\mathrm{g} / \mathrm{l}$ & $6,75 \pm 1,73^{a}$ & $5,88 \pm 1,09^{\mathrm{a}}$ & $6,35 \pm 1,18^{\mathrm{a}}$ \\
\hline & $\%$ & $8 \pm 2,16 \%{ }^{a}$ & $7,55 \pm 1,34^{\mathrm{a}}$ & $7,32 \pm 1,42^{\mathrm{a}}$ \\
\hline \multirow{2}{*}{$\gamma$-Globuline } & $\mathrm{g} / \mathrm{l}$ & $28,5 \pm 4,6^{\mathrm{a}}$ & $21,03 \pm 3,04^{b}$ & $24,62 \pm 3,27^{\mathrm{c}}$ \\
\hline & $\%$ & $33,53 \pm 5,67 \%^{\mathrm{a}}$ & $28,6 \pm 2,04^{b}$ & $30,32 \pm 3,63^{\mathrm{c}}$ \\
\hline \multicolumn{2}{|l|}{$\mathrm{A} / \mathrm{G}$} & $0,82 \pm 0,19^{\mathrm{a}}$ & $1,09 \pm 0,17^{b}$ & $0,90 \pm 0,09^{\mathrm{a}}$ \\
\hline
\end{tabular}


Tableau 3: Valeurs moyennes et proportions des différentes fractions protéiques chez les vaches Gobra ayant subi des pertes embryonnaires en fonction de la période d'apparition.

\begin{tabular}{|c|c|c|c|}
\hline $\begin{array}{l}\text { Paramètres } \\
\text { biochimiques }\end{array}$ & $\begin{array}{c}\text { MET } \\
(n=21)\end{array}$ & $\begin{array}{c}\text { MET J J } \mathbf{J}-\mathbf{J}_{35} \\
(\mathbf{n}=\mathbf{1 1})\end{array}$ & $\begin{array}{c}\text { MET J J } \mathbf{J}_{3-}-\mathbf{J}_{60} \\
(\mathrm{n}=10)\end{array}$ \\
\hline Albumine & $46,08 \pm 6,08 \%$ & $45,33 \pm 7,43 \%^{\mathrm{a}}$ & $46,91 \pm 4,42 \%^{\mathrm{a}}$ \\
\hline$\alpha 1-G l o b u l i n e$ & $2,76 \pm 1,01 \%$ & $2,56 \pm 0,840 \%^{\mathrm{a}}$ & $2,99 \pm 1,19 \%^{\mathrm{a}}$ \\
\hline$\alpha 2$-Globuline & $9,36 \pm 2,08 \%$ & $9,13 \pm 1,28 \%^{a}$ & $9,62 \pm 2,77 \%^{\mathrm{a}}$ \\
\hline$\beta$-Globuline & $8 \pm 2,16 \%$ & $7,62 \pm 2,72 \%^{\mathrm{a}}$ & $8,43 \pm 1,35 \%^{\mathrm{a}}$ \\
\hline$\gamma$-Globuline & $33,53 \pm 5,67 \%$ & $34,90 \pm 6,08 \%{ }^{\mathrm{a}}$ & $32,03 \pm 5,07 \%^{a}$ \\
\hline $\mathrm{A} / \mathrm{G}$ & $0,82 \pm 0,19$ & $0,81 \pm 0,21$ & $0,85 \pm 0,15$ \\
\hline
\end{tabular}

Les moyennes en ligne affectées de lettres différentes sont significativement différentes $(\mathrm{p}<0,05)$.

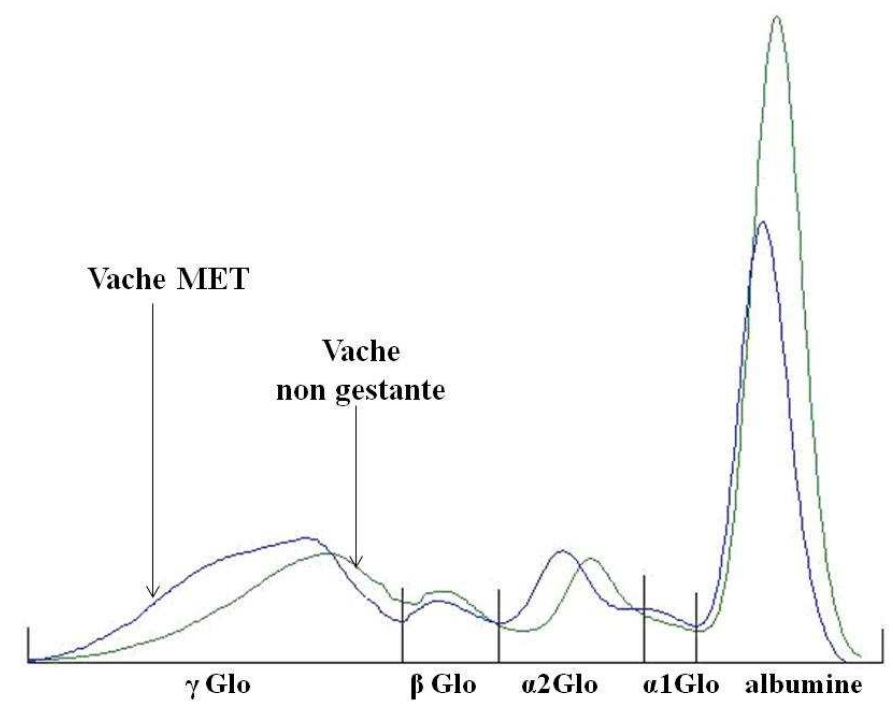

Figure 2: Profils électrophorétiques comparés de la vache ayant subi une mortalité embryonnaire (MET) et d'une vache non gestante.

\section{DISCUSSION}

Le taux de réussite de l'insémination a été de $47 \%$. Ce résultat est similaire à ceux qui ont été obtenus dans les programmes d'amélioration génétique au Sénégal (Kouamo et al., 2009 ; Kouamo et al., 2010), mais reste inférieur à l'optimum de $60 \%$ visé dans le programme d'insémination artificielle national (Kouamo et al., 2009). Sur les 43 vaches diagnostiquées non gestantes à $\mathbf{J}_{60}$ post IA, 21 ont été victimes des MET (26\%). Ce taux de mortalités embryonnaires est élevé car Santos et al. (2009) estimaient que, chez les vaches laitières, ce taux serait compris entre 5 et $12 \%$ selon les études. Silke et al. (2002) ont observé une perte du conceptus de $7,2 \%$ pour les vaches et de $6,1 \%$ chez les génisses entre les jours 28 et 
84 post gestation. Les facteurs génétique, endocrinien, immunologique, nutritionnelle, environnementale et pathologique pourraient être à l'origine de ce fort taux de MET (Chebel et al., 2004 ; Romano et al., 2007).

La protéinémie totale est l'un des paramètres biochimiques le moins significatif chez la vache. Elle est en effet dépendante des valeurs de l'albumine et des globulines totales. Elle sera donc basse ou élevée selon les concentrations de ces deux paramètres. Les valeurs usuelles de la concentration en protéines totales chez les vaches de race Gobra sont comprises entre 59 à $80 \mathrm{~g} / \mathrm{l}$ (Sawadogo et al., 1991). Les concentrations moyennes en protéines totales présentées dans le Tableau 2 ont montré que les groupes de vaches gestantes et des vaches ayant subi une MET ont une protéinémie statistiquement plus élevée à $\mathrm{J}_{35}$ que les vaches non gestantes $(p<0,05)$.

L'électrophorèse des protéines sériques donnant des informations supplémentaires sur les proportions des différentes fractions protéiques (Alberghina et al., 2011), il a été observé dans cette étude que le rapport albumine sur globuline $(\mathrm{A} / \mathrm{G})$ est significativement différent $(\mathrm{p}<0,05)$ entre les vaches non gestantes et celles ayant subi une perte embryonnaire. Ce rapport a été de 0,82 $\pm 0,19$ pour le groupe des vaches ayant subi une MET, ce qui traduit une proportion plus élevée de globulines que d'albumine chez ces vaches. Les vaches non gestantes ont en moyenne un rapport $\mathrm{A} / \mathrm{G}$ égal à $1,09 \pm 0,17$, ce qui signifie qu'en moyenne, il y'a sensiblement autant d'albumine que de globulines. L'analyse des profils électrophorétiques obtenus chez ces vaches permet de constater que même en cas de protéinémie normale, un dysfonctionnement au niveau de la répartition des différentes fractions protéiques peut survenir. Le dosage des protéines par électrophorèse est une méthode d'analyse qualitative des protéines, car toute interprétation d'un protéinogramme suppose en premier lieu la connaissance des variations physiopathologiques de la protéinémie (Carrer, 1998 ; Daunizeau, 2003).

Les différentes proportions de protéines obtenues dans cette étude chez les vaches gestantes sont comparables à celles qui ont été obtenues par Sawadogo (1987, 1992). Ces auteurs ont obtenu au Sénégal, chez des femelles zébu Gobra gestantes âgées de 2 - 3 ans, des proportions de protéines sériques de 45,2 \pm 4,2\% pour l'albumine, $14,25 \pm 1,3 \%$ pour les $\alpha$ globulines, $12,5 \pm 2,3 \%$ pour les $\beta$ globulines, $28,1 \pm 3 \%$ pour les $\gamma$-globulines, $0,82 \pm 0,06$ pour le rapport albumine sur globuline . Par contre, Gidel (1962) avait obtenu au Burkina Faso (ex Haute Volta), chez des zébus locaux, des proportions de protéines sériques de $41,3 \pm 2,9 \%$ pour l'albumine, $5,1 \pm 0,8 \%$ pour les $\alpha 1$ globulines, $8,3 \pm 0.56 \%$ pour les $\alpha 2$ globulines; $12,7 \pm 1,9 \%$ pour les $\beta$ globulines; $32,3 \pm 3 \%$ pour les $\gamma$-globulines; $0,72 \pm 0,08 \%$ pour le rapport A/G. La moyenne très faible d'albumine obtenue par Gidel (1962) comparée à celle de cette étude, pourrait s'expliquer par des techniques obsolètes d'électrophorèse qui ne permettaient pas une bonne migration des protéines en particulier les fractions d'albumine (Peltre, 1990). Chorfi et al. (2004) auraient constaté des différences significatives de profil électrophorétique chez la vache selon la méthode électrophorétique utilisée.

Les concentrations moyennes d'albumine n'ont pas montré de différences significatives en fonction de l'état physiologique, bien que, la proportion en albumine chez les vaches non gestantes soit statistiquement élevée $(\mathrm{p}<0,05)$. Cette variation de proportion d'albumine s'expliquerait par la variation des concentrations en globulines (Carrer, 1998). Les variations de l'albuminémie seraient le plus souvent dues à l'alimentation ou à un dysfonctionnement hépatique. L'albumine étant synthétisée par le foie à partir des protéines absorbées dans l'intestin et des protéines corporelles. La concentration 
d'albumine dans le sang est donc directement fonction de la différence entre les apports alimentaires et les prélèvements corporels (Rowlands and Manston ,1983).

Le rapport $\mathrm{A} / \mathrm{G}$ dans les groupes de gestantes et de MET sont en faveur des globulines. Les globulines sont synthétisées par les lymphocytes et les plasmocytes. La concentration en globulines apporterait donc des informations sur le statut humoral et l'état immunitaire des vaches, une composante du système de défense de l'organisme (Chorfi et al., 2004). L'élévation de la concentration des globulines pourrait être la conséquence d'un processus infectieux ou inflammatoire aigu ou chronique (Brugére-Picoux and Remy, 1995; Sattler, 2003). Par ailleurs, il existerait une relation entre l'augmentation de la concentration en globulines et la fertilité des vaches (Rowlands et al., 1977). L'augmentation significative des $\alpha 1$-globulines chez les vaches gestantes, serait due à l'ensemble des phénomènes inflammatoires qui sont mis jeu en début de gestation. Ce résultat confirme celui de Zvorc et al. (2000) qui avaient observé une augmentation des $\alpha$-globulines au cours de la gestation. Par contre, Cairoli et al. (2006) avaient constaté une augmentation des $\alpha$-globulines chez des vaches à inséminer atteintes des endométrites post-partum.

Les moyennes de proportions de $\gamma$ globulines ont été élevées dans le groupe des vaches ayant subi une MET par rapport aux moyennes des autres groupes de vaches (gestantes et non gestantes). L'un des intérêts clinique de la fraction des $\gamma$-globulines est que les immunoglobulines migrent essentiellement dans la zone des $\gamma$ globulines. Ainsi, la fraction $\gamma$-globuline représente les protéines de l'immunité. L'augmentation de cette fraction de $\gamma$ globuline chez les vaches peut être due à des problèmes infectieux (Abenga and Anosa, 2005 ; Yameogo et al., 2008 ; Apaydin and Dede, 2010), qui peuvent être la cause probable de ces ME. Dans cette étude, la période de survenue des $\operatorname{MET}\left(\mathrm{J}_{21}-\mathrm{J}_{35}\right.$ et $\mathrm{J}_{35}$ $\mathrm{J}_{60}$ ) n'a pas eu une influence sur le profil électrophorétique ; cela pourrait s'expliquer par la courte différence de temps ( $>25$ jours) entre ces deux périodes de pertes embryonnaires. Zvorc et al. (2000) par contre, avaient constaté que le stade de gestation influence le protéinogramme chez les vaches.

\section{Conclusion}

D'après nos résultats, nous avons observé que le rapport albumine sur globuline a été significativement différent entre les vaches suivant leur statut physiologique. Chez les vaches ayant subi des pertes embryonnaires, les fractions de $\gamma$ globulines ont été élevées. Ainsi donc, des causes infectieuses de ces avortements peuvent être suspectées. Un accent particulier doit être mis pour rechercher les causes des MET afin de pouvoir les éradiquer et limiter les pertes économiques dans les productions animales car le coût de l'insémination artificielle reste élevé pour l'éleveur africain.

\section{REMERCIEMENTS}

Nous remercions l'Agence Universitaire de la Francophonie (AUF) et le Programme National d'Insémination Artificielle (PNIA) pour leur soutien à ces travaux de recherche. Nos remerciements vont également à l'endroit des éleveurs, des inséminateurs et de tout le personnel du Laboratoire de Biochimie de l'EISMV de Dakar pour leur disponibilité.

\section{REFERENCES}

Abenga JN, Anosa VO. 2005. Serum total proteins and creatinine levels in experimental Gambian trypanosomosis of vervet monkeys. Afr. J. Biotech., 4: 187-190.

Alberghina D, Giannetto C, Vazzana I, Ferrantelli V, Piccione G. 2011. Reference intervals for total protein concentration, serum protein fractions, and albumin/globulin ratios in clinically healthy dairy cows. J. Vet. Diagn. Invest., 23: 111-114. 
Apaydin B, Dede S. 2010. Profil électrophorétique des protéines sériques de moutons naturellement infectés par Babesia ovis. Rev. Méd. Vét., 161(2): 5760 .

Bajaj NK, Sharma N. 2011. Endocrine Causes of Early Embryonic Death: An Overview. Curr. Res. Dairy Sci., 3: 1-24.

Brugere-Picoux J, Remy D. 1995. Biochimie clinique. Maladies métaboliques chez la vache laitière, La Dépêche Technique, 46 : 26-29.

Cairoli F, Battocchio M, Veronesi MC, Brambilla D, Conserva F, Eberini I, Wait R, Gianazza E. 2006. Serum protein pattern during cow pregnancy: Acutephase proteins increase in the peripartum period. Electrophoresis, 27: 1617-1625.

Carrer Le D. 1998. Electrophorèse et Immunofixation des Protéines Sériques: Interprétations Illustrées. Laboratoire SEBIA ; p.122.

Chebel RC, Santos JEP, Reynolds JP, Cerri RLA, Juchem SO, Overton M. 2004. Factors affecting conception rate after artificial Insemination and pregnancy loss in lactating dairy cows. Anim. Reprod. Sci., 84: 239-255.

Chorfi Y, Lanevschi-Pietersma A, Girard V, Tremblay A. 2004. Evaluation of variation in serum globulin concentrations in dairy cattle. Vet. Clin. Pathol., 33(3): 122-7.

Daunizeau A. 2003. Imunoglobines Monoclonales. Cahier de Formation Biologie Médicale, 28: 26-33.

Dunne LD, Diskin MG, Sreenan JM. 2000. Embryo and foetal loss in beef heifers between day 14 of gestation and full term. Anim. Reprod. Sci., 58: 39-44.

Gidel R. 1962. Etude électrophorétique quantitative en gelose des protéines sériques de bovins. Rev. Elev. Med. Pays Trop., 15 : 259-263.

Inskeep EK, Dailey RA. 2005. Embryonic death in cattle. Vet. Clin. North Am. Food Anim. Pract., 21: 437-461.

Kouamo J, Sow A, Leye A, Sawadogo GJ, Ouédraogo GA. 2009. Amélioration des performances de production et de reproduction des bovins par l'utilisation de l'insémination artificielle en Afrique Sub-saharienne et au Sénégal en particulier: état des lieux et perspectives. RASPA, 7: 139-148.

Kouamo J, Habimana S, Alambedji BR, Sawadogo GJ, Ouedraogo GA. 2010. Séroprévalence de la brucellose, de l'IBR et de la BVD et impact sur la reproduction des femelles zébus Gobra et croisements inséminées en milieu traditionnel dans la région de Thiès au Sénégal. Rev. Méd. Vét., 161(7): 314321.

Peltre G. 1990. Electrophorèse, les trois principes de base. Technique et Biologie, 1: $16-23$.

Ponsart C, Dubois P, Charbonnier G, Leger T, Freret S, Humblot P. 2007. Evolution de l'état corporel entre 0 et 120 jours de lactation et reproduction des vaches laitières hautes productrices. Journées nationales des GTV. Nantes, 347-356.

Romano JE, Thompson JA, Kraemer DC, Westhusin ME, Forrest DW, Tomaszweski MA. 2007. Early pregnancy diagnosis by palpation per rectum: Influence on embryo/fetal viability in dairy cattle. Theriogenology, 67: 486-493.

Rowlands GJ, Little W, Kitchenham BA. 1977. Relationship between blood composition and fertility in dairy cows a field study. J. Dairy Sci., 44: 1-7.

Rowlands GJ, Manston R. 1983. Decline of serum albumin concentration at calving in dairy cows: its relationships with age and association with subsequent fertility. Res. Vet. Sci., 34: 90-96.

Santos JEP, Rutigliano HM, Sa Filho MF. 2009. Risk factors for resumption of postpartum estrous cycles and embryonic survival in lactating dairy cows. Anim. Reprod. Sci., 110: 207-221.

Sattler N. 2003. Intérêts et limites des analyseurs en buiatrie. Le Point Vét. Num. Spé., 34: 32-35. 
Sawadogo GJ. 1987. Protéines sériques totales et fractions chez le zebu Gobra du Senegal: effets de l'âge et du sexe. Rev. Méd. Vét., 138: 493-497.

Sawadogo GJ, Oumarou AA, Sene M, Diop M. 1991. Effects of poor pasture conditions and type of feeding on some biochemical values of Gobra zebu in Senegal. Brit. Vet. J., 147(6): 538-544.

Sawadogo GJ, Abouna A, Hamadama H, Maikano A. 1992. Principaux minéraux et protéines sériques et fractions chez le zébu Goudali du Cameroun septentrional. Rev. Méd. Vét., 142(6): 493-497.

Silke V, Diskin MG, Kenny DA, Boland MP, Dillon P, Mee JF, Sreenan JM. 2002. Extent, pattern and factors associated with late embryonic loss in dairy cows. Anim. Reprod. Sci., 71: 1-12.

Sousa NM, Zongo M, Pitala W, Boly H, Sawadogo L, Sanon M, Figueiredo JR, Goncalves PBD, El Amiri B, Perényi Z, Beckers JF. 2003. Pregnancy-associated glycoprotein concentrations during pregnancy and the postpartum period in Azawak zebu cattle. Theriogenology, 59: 1131-1142.

Tietz. 1994. Textbook of Clinical Chemistry ( $2^{\text {nd }}$ edn). Burtis CA, Ashwood ER (eds). WB Saunders Co.

Vall E, Bayala I. 2004. Note d'état corporel des zébus soudanais. In Production animale en Afrique de l'Ouest; fiche technique $\mathrm{N}^{\circ} 12$, p.8.

Yameogo N, Ouedraogo GA, Kanyandekwe C, Sawadogo GJ. 2008. Relationship between ketosis and dairy cows' blood metabolites in intensive production farms of the periurban area of Dakar. Trop Anim. Health Prod., 40: 483-490.

Zoli AP, Guilbault LA, Delahaut P, Benitez OW, Beckers JF. 1992. Radioimmunoassay of a bovine pregnancy-associated glycoprotein in serum: its application for pregnancy diagnosis. Biol. Reprod., 46: 83-92.

Zvorc Z, Matijatko V, Beer B, Foršek J, Bedrica L, Kucer N. 2000. Blood serum proteinograms in pregnant and nonpregnant cows. Vet. Archiv., 70: 21-30. 